\section{ACUTE INJURIES OF THE HEAD 2nd edition}

By G. F. Rowbottham. Published by E. \& S. Livingstone, Edinburgh. I946. Price 30s. net.

The second edition of this book has made its appearance within three years and this fact by itself is sufficient proof of its popularity. It is a brilliant book and one which should be read by both surgeon and physician. It deals very conclusively with all forms of head injuries and discusses with clarity the pathology, anatomy and diagnosis of injuries of the head. It is beautifully illustrated throughout and is written with great simplicity and directness. The format is extremely good and the pictures simple yet explicit; indeed, many of the illustrations are very beautiful. The publishers, too, are to be congratulated on the production of a book which will for a long time be considered one of the classics of neuro-surgery.

\section{EXTENSILE EXPOSURE APPLIED TO LIMB SURGERY 127 illustrations}

By Arnold K. Henry, M.B., M.Ch., F.R.C.S.I. Published by E. \& S. Livingstone, Ltd. (Sept., I945). Price 3os. net.

This book lives up to the high standard which the publishers have set themselves, and the author is to be congratulated upon presenting a text so full of wisdom and illustrated by so many excellent figures. Many experienced surgeons will read this book with profit, and certainly all budding surgeons must read it. Postgraduates will do well to mark well and inwardly digest it, for this book of some $\mathbf{1 7 0}$ pages has within its covers much that will help the everyday life of the would-be surgeon. It is very highly recommended.

\section{THE ELEMENTS OF MEDICAL TREATMENT 4th edition}

By Sir Robert Hutchison, Bart. Bristol: John Wright \& Sons, Ltd. London: Simpkin Marshall (194r), Ltd., 1945. Price Ios. 6d.

To review Hutchison's Elements of Medical Treatment is as presumptuous and unnecessary as to take the proverbial ham-sandwich to the Lord Mayor's Banquet. Since its first publication in 1926 this little manual has rapidly become a medical classic and a best-seller. "Largely designed to instruct the student in the fast disappearing art of prescribing," though scrupulously revised and thoroughly modernised-this edition contains a new chapter on chemotherapy with a page devoted to penicillin -its original purpose and scope remain unchanged. Practical and sober in outlook, lucid and intimate in presentation, the Elements, in addition to being indispensable for reference, make delightful reading. They bristle with aphorisms ("one must cut one's therapeutic coat according to one's cloth") and abound in typical Hutchisonian touches, such as recommending the novels of Anthony Trollope as a suitable mental pabulum for the patient with insomnia.

Dr. S. L. Simpson has rewritten Chapter XX on Endocrine treatment, in which he briefly refers to the synthetic oestrogens and to thiouracil.

There is a useful index (after "foam baths" "I53" should read " 173 "), but we are disappointed in the appearance of the title-page.
THE INFANT: A HANDBOOK OF MANAGEMENT 3rd edition

By W. J. Pearson and A. G. Watkins. H. K. Lewis \& Co., Ltd., London. I945. Price 4s.

We are really glad to welcome a third edition of this deservedly popular handbook, which includes two new sections: preventive inoculation and tables of vitamin requirements, values and sources. Intended for "Infant Welfare workers (lay as well as medical), house physicians, medical students, pupil midwives, and last but not least, the general practitioner," and containing "information of a kind hitherto only available by selection from the numerous large text-books on the subject," this little manual remains concise, readable, and easy to refer to despite the absence of an index.

We should be grieved if one day the Infant were to grow into a bulkier size.

\section{A COMPLETE OUTLINE OF FRACTURES}

By J. Grent Bonnin, M.D., B.S., F.R.C.S. 2nd edition. Published by Wm. Heinemann (Medical Books) Limited, London, 1946.

This textbook on fractures is already well known, but several chapters in the New Edition have been brought up to date and the reader will find most useful the chapter dealing with wounds and compound fractures, in which the author has dealt at length with the newer antiseptics, including penicillin. This book should certainly be in the hands of all resident doctors handling fractures, though its use by the senior members of the profession may be somewhat restricted by a certain incompleteness on difficult points of treatment; the reviewer had a need recently form guidance as to a particular line of treatment for a difficult fracture, but on referring to the book found the advice given was merely to operate. The diction also is rather involved at times and needs careful following. The X-rays which are numerous, would perhaps have been reproduced better if done on a shiny surface, but these defects are remedied by the excellent line drawings which amply? illustrate the book.

\section{THE PROBLEM OF LUPUS VULGARIS}

By Robert Aitken, M.D., F.R.C.P.E., F.R.C.E. E. \& S. Livingstone, Ltd. Pp. 66. Price I5s.

Dr. Aitken has written a most interesting and readable little book about Lupus, well illustrated with many plates in colour and black and white.

He explains in simple terms the evolution and method of spread of the disease, and shows how the face is most commonly affected, and how rarely the condition is associated with phthisis, although more commonly with bone and gland tuberculosis. He demonstrates that the disease often begins in childhood or early adolescenceindeed more than half the cases began during the child's school life.

The chapter on aetiology establishes the tuberculous nature of the disease and records cases of direct inoculation with the tubercle bacillus, while that on treatment stresses the importance of general treatment and explains amply the classical destructive therapies. Dr. Aitken rightly condemns the use of X-rays, but the reviewer's personal experience with the hydnocarpate esters has been satisfactory.

There is a detailed chapter on the use of ultra-violet irradiation, explaining the reason for the author's strong preference for the carbon-arc lamp, and a very interesting 
chapter on the history and use of tuberculin in Lupus Vulgaris with a warning against the use of general ultraviolet light and tuberculin in the presence of pulmonary tuberculosis. Dr. Aitken is much concerned with the public health and social aspects of the disease and suggests the formation of regional Lupus centres.

It is to be hoped that the recent remarkable work with Calciferol will render Lupus a much more curable and less dreaded disease than it has been, and it is a pity that the book was published just too soon to mention the new treatment.

\section{THE SYMPTOMATIC DIAGNOSIS AND TREAT- MENT OF GYNAECOLOGICAL DISORDERS}

By Margaret Moore White. Lewis, London.

Many authors who write for the practising doctor get so caught up in a mass of detail that their books completely fail in their purpose. Not so with Miss Margaret Moore White who has supplied us with a book, not too voluminous, which is eminently suitable for the needs of the general practitioner.

The necessary essentials are concisely dealt with and the doctor who wishes to treat a case of Pruritis, or to interpret pelvic pain, need only refer to the appropriate sections to obtain the information he wants. Miss Moore White has mastered the technique of saying what is necessary and saying it clearly and concisely.

I was particularly struck by the chapter on Contraception. This is a more able treatise than one finds in many larger and more authoritative books. I felt that stress should be laid on the fact that the fitting of contraceptive appliances is highly specialised work and, in my opinion, should be carried out at Clinics. Appliances fitted by comparatively inexpert people so often result in tragic pregnancies. This contraceptive section is written by Miss Mary Redding.

The discussion on pre-operative and post-operative treatment of cases will be a boon to many readers.

\section{NEUROSIS AND THE MENTAL HEALTH SERVICES}

By C. P. Blacker. Foreword by Sir Wilson Jameson. Published by Humphrey Milford, Oxford University Press, London. 1946. Pp. 218. Price 2 Is.

This monumental and stimulating work, at the same time far-sighted and meticulously detailed, consists of an introduction dealing with the origins, history, and scope of the survey, sponsored in the first instance by the Ministry of Health; four principal parts: main findings and their interpretation, the mental health services of the future, psychiatric services of the post-war transitional period, findings of the survey in a series of tables; and seven appendices. Much of the material here presented makes interesting reading, while other sections are useful for reference. The author discusses among others the partial insulation of psychiatry from general medicine, the training of psychiatrists and of mental nurses, the Medical Officer of Mental Health, neurosis in industry, voluntary sterilization in the prevention of mental infirmities, and child guidance centres, psychiatric clinics, and hostels for unstable or difficult children. The book is written in a style refreshingly free of "psychiatric jargon than which none is more exasperating to the general reader nor more inimical to clear thinking." The Index must have been most difficult to compile. But we must confess we do not like the gilt lettering on the spine of the volume.

NOTABLE NAMES IN MEDICINE AND SURGERY By Hamilton Bailey and W. J. Bishop. Published by H. K. Lewis \& Co., Ltd., London. I946. Yp. 202. 238 illustrations. Price I5s.

We welcome the appearance of a second edition of this most useful, delightful, and scholarly book which now carries the cumbersome sub-title "Short biographies of some of those whose discoveries (not necessarily the greatest medical discoveries) have become eponymous in the medical and allied professions." Some of the photographs from the first edition have been retouched, but have, unfortunately, deteriorated in the process. It is pointed out that Bromley Church with the tablet to John Scott's memory no longer exists. The reviewer always understood that Pirquet committed suicide. For their forthcoming book More Notable Names the compilers might consider quoting from the original description of a discovery, since eponymously commemorated. It is felt that a note on "Further Reading" at the end of each entry might be appreciated by many readers. To suggest that not all the biographies have the same succintly intimate characterization as, for example, Murphy and Welch, implies a note, not of criticism, but of regret. No teacher, student, and doctor should be without this book which is remarkably moderate in price considering its wealth of illustrations, and has a good index.

\section{DOCTOR IN THE MAKING: THE ART OF} BEING A MEDICAL STUDENT

By Arthur W. HaM, M.B., and M. D. Salter, M.A., Ph.D. Medical Publications, Ltd., London, 1944. Price 9s. 6d. net.

This book is a very interesting discussion of the medical student course, by an associate Professor of Anatomy and a Lecturer and Research Fellow in the Department of Psychology, both in the University of Toronto. Starting from the motives which influence the choice, and the equipment necessary to make a successful student, the analysis and synthesis proceeds through eleven chapters in considering such subjects as "Organising your knowledge," "Memory versus Understanding," "Forgetting," "Your Child-self," "Understanding and Applying the Scientific Method," and "Do's and Don'ts for the Student." It actually .discusses how "Professor $\mathrm{X}$ believes himself to be an essentially kindly soul, but is greatly irritated by stupidity. . . . Actually, Professor $\mathrm{X}$ is frustrated in his desire to be important. This makes him irritable, and he vents his emotion in sarcastic outbursts directed at his students. He feels more important after making his students appear confused and awkward. . . ." So there you have the reason for the kind of conduct some of us have suffered from.

The book is one that could well be distributed by the Seats of Learning to their Neophytes in all Faculties, as they arrive to start their gruelling courses of study. 